\title{
Five New Withanolides from Tacca plantaginea
}

\author{
Hai-Yang Liv, ${ }^{a}$ Wei Ni, ${ }^{a}$ Bai-Bo XIE,,${ }^{a}$ Ling-Yun Zhou, ${ }^{a}$ Xiao-Jiang HaO,${ }^{a}$ Xin WAnG,,${ }^{b}$ and \\ Chang-Xiang $\mathrm{CHEN}^{*, a}$ \\ ${ }^{a}$ State Key Laboratory of Phytochemistry and Plant Resources in West China, Kunming Institute of Botany, Chinese \\ Academy of Sciences; Kunming 650204, Yunnan, People's Republic of China: and ${ }^{b} 4060$ Group, Institute of Chemistry, \\ Chinese Academy of Sciences; Beijing 100080, People's Republic of China. \\ Received January 24, 2006; accepted April 19, 2006
}

Five new withanolides named plantagiolides $A-E(1,3-6)$, together with a known withanolide glucoside, chantriolide A (2) were isolated from the whole plants of Tacca plantaginea (HANCE). Their structures were elucidated by means of spectroscopic methods including extensive 1D and 2D-NMR techniques.

Key words Tacca plantaginea; plantagiolide A; plantagiolide B; plantagiolide C; plantagiolide D; plantagiolide E

Taccaeae, a small family, includes Tacca and Schizacapsa genus and is predominately distributed in the tropical region of Asia, the Pacific Islands, and Australia. ${ }^{1)}$ Taccalonolides, ${ }^{2-4)}$ anthocyanins, ${ }^{5)}$ diarylheptanoids and diarylheptanoid glucosides, $\left.{ }^{6}\right)$ steroidal sapogenins and steroidal glycosides such as C-27 steroidal saponins, C-28 sterol glucosides and withanolide glucosides ${ }^{7-15)}$ have been isolated from some Tacca species.

Tacca plantaginea (HANCE) is a perennial plant that grows in southeastern China. Its rhizomes is a folk medicine used as analgesic, antipyretic, anti-inflammatory agents and for the treatment of incised wounds. ${ }^{16)}$ Chen and co-workers have reported the isolation and purification of the highly oxygenated taccalonolides from the rhizomes of $T$. plantaginea. ${ }^{17-21)}$ Taccalonolides $\mathrm{A}$ and $\mathrm{E}$ were claimed to be important for initiation of paclitaxel-like microtubule bundling; therefore, they represent the first plantderived microtubulestabilising agents to be identified since paclitaxel and the first natural steroids to show microtuble-stabilizing activity. ${ }^{22)}$ Previously, we have reported two new steroidal saponins from this whole plant. ${ }^{23}$ Further chemical investigation on the $\mathrm{CHCl}_{3}$ soluble part of the EtOH extracts of this plant resulted in the isolation of five new withanolides, named plantagiolides $\mathrm{A}-\mathrm{E}(\mathbf{1}, \mathbf{3}-\mathbf{6})$. The known chantriolide A (2) was separated from the AcOEt soluble part. The structures of the five new compounds were determined by analysis of their spectral data, especially two dimensional (2D) NMR spectra. Herein, we reported the isolation and structural elucidation of those compounds.

Plantagiolide A (1) was obtained as colorless needles. Its molecular formula of $\mathrm{C}_{32} \mathrm{H}_{42} \mathrm{O}_{11}$ was established by the positive high-resolution electrospray ionization mass spectrum $(\mathrm{HR}-\mathrm{ESI}-\mathrm{MS})\left([\mathrm{M}+\mathrm{Na}]^{+}, m / z \quad 625.2623\right)$ and ${ }^{13} \mathrm{C}-\mathrm{NMR}$

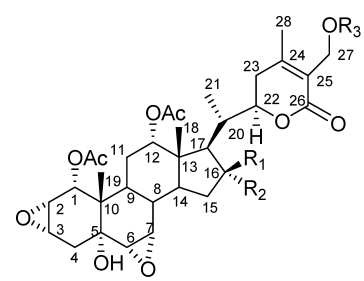

$1 \quad \mathrm{R}_{1}+\mathrm{R}_{2}=\mathrm{O}, \mathrm{R}_{3}=\mathrm{H}$

$2 R_{1}+R_{2}=O, R_{3}=$ glc.py

$3 \quad \mathrm{R}_{1}=\mathrm{OH}, \mathrm{R}_{2}=\mathrm{R}_{3}=\mathrm{H}$

$4 \quad \mathrm{R}_{1}=\mathrm{R}_{2}=\mathrm{R}_{3}=\mathrm{H}$

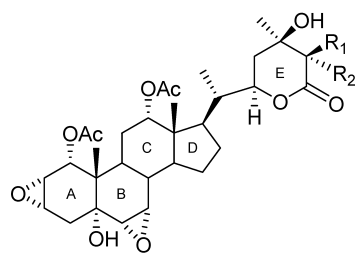

$5 \mathrm{R}_{1}=\mathrm{CH}_{3}, \mathrm{R}_{2}=\mathrm{H}$ $6 \mathrm{R}_{1}=\mathrm{OH}, \mathrm{R}_{2}=\mathrm{CH}_{3}$ spectrum (Table 2), a molecular formula corresponding to 12 degrees of unsaturation. A careful comparison the ${ }^{1} \mathrm{H}$ - and ${ }^{13} \mathrm{C}$-NMR data of $\mathbf{1}$ with those of the aglycone of chantriolide A (2), ${ }^{13)}$ it was obvious that compound $\mathbf{1}$ was the aglycone of chantriolide A (2) and the stereochemistry of compound 1 was same as that of the aglycone of chantriolide A (2). Accordingly, the structure of $\mathbf{1}$ was elucidated as $(20 S, 22 R)$ $1 \alpha, 12 \alpha$-diacetoxy- $2 \alpha, 3 \alpha ; 6 \alpha, 7 \alpha$-diepoxy-5 $\alpha, 27$-dihydroxy16-oxowith-24-enolide, named plantagiolide A, which was firstly obtained as natural compound.

Plantagiolide B (3), a white amorphous powder, showed a molecular ion peak at $m / z 605[\mathrm{M}+\mathrm{H}]^{+}$in its positive fast atom bombardment mass spectrometry (FAB-MS), consistent with a molecular formula $\mathrm{C}_{32} \mathrm{H}_{44} \mathrm{O}_{11}(11$ degrees of unsaturations), as confirmed by its (HR)-ESI-MS ([M+Na $]^{+}, m / z$ 627.2776) and ${ }^{13} \mathrm{C}$-NMR spectrum. By step-by-step comparison of the ${ }^{1} \mathrm{H}$ - and ${ }^{13} \mathrm{C}$-NMR spectral features of $\mathbf{3}$ with those of $\mathbf{1}$, the other signals of $\mathbf{3}$ were similar to those of $\mathbf{1}$ except for the disappearance of a carbonyl group and the presence of a hydroxyl group located at C-16 $(\delta$ 70.6) and an oxygenated methine $(\delta$ 4.34) in compound 3. Therefore, it was supposed that the carbonyl group at $\mathrm{C}-16$ was protonated to hydroxyl group in compound $\mathbf{3}$, which was confirmed by the mass difference of $m / z=2$ and the heteronuclear multiple bond correlation (HMBC) spectrum. In the HMBC spectrum of 2, the correlations of H-14 $(\delta 2.54, \mathrm{dd}, J=8.0,10.0 \mathrm{~Hz})$, $\mathrm{H}_{\alpha}-15(\delta 2.50, \mathrm{~m}), \mathrm{H}_{\beta}-15(\delta 1.40, \mathrm{~m}), \mathrm{H}-17(\delta 1.64, \mathrm{~m})$, and $\mathrm{H}-20(\delta 2.36, \mathrm{~m})$ with $\mathrm{C}-16(\delta 70.6, \mathrm{~d})$ were observed. The relative configuration of the hydroxyl group in $\mathrm{C}-16$ was determined by ROESY experiment (Fig. 1). The H-16 signal showed ROESY correlations with $\mathrm{H}-14$ and $\mathrm{H}-17$ suggesting a $\alpha$-orientation of $\mathrm{H}-16$. On the basis of above evidence, the structure of 3 was established as $(20 S, 22 R)-1 \alpha, 12 \alpha$-diacetoxy- $2 \alpha, 3 \alpha ; 6 \alpha, 7 \alpha$-diepoxy-5 $\alpha, 16 \beta, 27$-trihydroxywith-24enolide, named plantagiolide $\mathrm{B}$, which was the aglycone of chantriolide $\mathrm{B}^{13)}$

Plantagiolide C (4) was isolated as colorless needles. The (HR)-ESI-MS of 3 displayed a $[\mathrm{M}+\mathrm{Na}]^{+}$peak at $\mathrm{m} / z$ 611.2835 , corresponding to the empirical molecular formula of $\mathrm{C}_{32} \mathrm{H}_{44} \mathrm{O}_{10}$, which was also deduced by analysis of its ${ }^{13} \mathrm{C}$ NMR and DEPT spectral data. The mass spectrum indicated that compound $\mathbf{4}$ was 14 mass units less than compound $\mathbf{1}$. The ${ }^{1} \mathrm{H}$ - and ${ }^{13} \mathrm{C}$-NMR spectral data of $\mathbf{4}$ were close to those of 1. The only significant differences included the absence 
Table 1. ${ }^{1} \mathrm{H}-\mathrm{NMR}$ Data for Compounds $\mathbf{1}, \mathbf{3}-\mathbf{6}(\delta$ in ppm; $J$ in $\mathrm{Hz})$

\begin{tabular}{|c|c|c|c|c|c|}
\hline $\mathrm{H}$ & $\mathbf{1}^{a, d)}$ & $\mathbf{3}^{a, e)}$ & $4^{a, d)}$ & $5^{c, e)}$ & $6^{b, e)}$ \\
\hline 1 & $4.65(\mathrm{~d}, 5.5)$ & $4.62(\mathrm{~d}, 5.2)$ & $4.54(\mathrm{~d}, 4.8)$ & $4.47(\mathrm{~d}, 5.2)$ & $4.86(\mathrm{~d}, 5.2)$ \\
\hline 2 & $3.75(\mathrm{dd}, 4.0,4.5)$ & $3.73(\mathrm{dd}, 4,0,4.6)$ & $3.65(\mathrm{brs})$ & $3.56(\mathrm{dd}, 4.1,4.6)$ & $3.89(\mathrm{dd}, 4.2,4.3)$ \\
\hline 3 & $3.45(\mathrm{brs})$ & $3.55(\mathrm{dd}, 1.6,3.3)$ & $3.48(\mathrm{brs})$ & $3.41(\mathrm{brd}, 3.0)$ & 3.64 (brs) \\
\hline $4 \alpha$ & $2.43(\mathrm{~d}, 16.0)$ & $2.41(\mathrm{~m})$ & $2.31(\mathrm{~d}, 15.6)$ & $2.21(\mathrm{~d}, 15.6)$ & $2.36(\mathrm{~m})$ \\
\hline $4 \beta$ & $2.10(\mathrm{~m})$ & $2.05(\mathrm{~m})$ & $2.00(\mathrm{~m})$ & $1.94(\mathrm{~d}, 6.1)$ & $2.20(\mathrm{~m})$ \\
\hline 6 & $2.90(\mathrm{~d}, 3.0)$ & $2.85(\mathrm{~d}, 3.5)$ & $2.77(\mathrm{~d}, 2.6)$ & $2.70(\mathrm{~d}, 3.4)$ & $3.02(\mathrm{~d}, 3.4)$ \\
\hline 7 & $3.09(\mathrm{br} \mathrm{s})$ & $3.12(\mathrm{brs})$ & $3.04(\mathrm{brs})$ & $2.97(\mathrm{brs})$ & $3.14(\mathrm{brs})$ \\
\hline 8 & $1.96(\mathrm{~m})$ & $1.83(\mathrm{~m})$ & $1.69(\mathrm{~m})$ & $1.63(\mathrm{~m})$ & $1.71(\mathrm{~m})$ \\
\hline 9 & $2.23(\mathrm{~m})$ & $2.04(\mathrm{~m})$ & $1.91(\mathrm{~m})$ & $1.85(\mathrm{~m})$ & $2.36(\mathrm{~m})$ \\
\hline $11 \alpha$ & $1.64(\mathrm{dd}, 3.5,14.5)$ & $1.56(\mathrm{~m})$ & $1.50(\mathrm{~m})$ & $1.73(\mathrm{~m})$ & $1.67(\mathrm{~m})$ \\
\hline $11 \beta$ & $1.61(\mathrm{~m})$ & $1.50(\mathrm{~m})$ & $1.44(\mathrm{~m})$ & $1.27(\mathrm{~m})$ & $1.33(\mathrm{~m})$ \\
\hline 12 & $5.00(\mathrm{brs})$ & 4.95 (brs) & 4.89 (brs) & 4.80 (brs) & $5.01(\mathrm{brs})$ \\
\hline 14 & $2.54(\mathrm{dd}, 8.0,10.0)$ & $1.96(\mathrm{~m})$ & $1.93(\mathrm{~m})$ & $1.84(\mathrm{~m})$ & $2.07(\mathrm{~m})$ \\
\hline $15 \alpha$ & $2.49(\mathrm{~d}, 8.5)$ & $2.50(\mathrm{~m})$ & $1.82(\mathrm{~m})$ & $1.72(\mathrm{~m})$ & $1.82(\mathrm{~m})$ \\
\hline $15 \beta$ & $2.04(\mathrm{~m})$ & $1.40(\mathrm{~m})$ & $1.26(\mathrm{dd}, 3.4,7.9)$ & $1.17(\mathrm{~m})$ & $1.25(\mathrm{~m})$ \\
\hline $16 \alpha$ & - & $4.34(\mathrm{~m})$ & $1.69(\mathrm{~m})$ & $1.44(\mathrm{~m})$ & $1.69(\mathrm{~m})$ \\
\hline $16 \beta$ & - & - & $1.35(\mathrm{~m})$ & $1.27(\mathrm{~m})$ & $1.24(\mathrm{~m})$ \\
\hline 17 & $2.53(\mathrm{~m})$ & $1.64(\mathrm{~m})$ & $1.67(\mathrm{~m})$ & $1.55(\mathrm{~d}, 9.1)$ & $1.83(\mathrm{~m})$ \\
\hline 18 & $1.01(\mathrm{~s})$ & $1.05(\mathrm{~s})$ & $0.74(\mathrm{~s})$ & $0.64(\mathrm{~s})$ & $0.69(\mathrm{~s})$ \\
\hline 19 & $0.85(\mathrm{~s})$ & $0.81(\mathrm{~s})$ & $0.74(\mathrm{~s})$ & $0.65(\mathrm{~s})$ & $0.81(\mathrm{~s})$ \\
\hline 20 & $2.36(\mathrm{~m})$ & $2.48(\mathrm{~m})$ & $1.86(\mathrm{~m})$ & $1.81(\mathrm{~m})$ & $2.05(\mathrm{~m})$ \\
\hline 21 & $0.95(\mathrm{~d}, 7.0)$ & $0.92(\mathrm{~d}, 6.9)$ & $0.80(\mathrm{~d}, 6.4)$ & $0.70(\mathrm{~d}, 6.9)$ & $1.11(\mathrm{~d}, 6.5)$ \\
\hline 22 & $4.97(\mathrm{~m})$ & $4.65(\mathrm{~m})$ & $4.36(\mathrm{~m})$ & $4.57(\mathrm{dt}, 3.1,6.3,12.0)$ & $5.14(\mathrm{brd}, 12.0)$ \\
\hline $23 \alpha$ & $2.34(\mathrm{~m})$ & $2.49(\mathrm{~m})$ & $2.37(\mathrm{~m})$ & $1.58(\mathrm{~m})$ & $2.80(\mathrm{brs})$ \\
\hline $23 \beta$ & $2.14(\mathrm{~m})$ & $2.13(\mathrm{~d}, 2.8)$ & $2.33(\mathrm{~m})$ & $1.44(\mathrm{~m})$ & $1.82(\mathrm{~m})$ \\
\hline 25 & - & - & - & $2.13(\mathrm{~d}, 7.1)$ & - \\
\hline $27 \mathrm{a}$ & $4.37(\mathrm{ABd}, 12.5)$ & $4.38(\mathrm{ABd}, 12.2)$ & $4.32(\mathrm{ABd}, 12.6)$ & $1.12(\mathrm{~d}, 7.1)$ & $2.05(\mathrm{~s})$ \\
\hline $27 \mathrm{~b}$ & $4.32(\mathrm{ABd}, 12.5)$ & $4.31(\mathrm{ABd}, 12.2)$ & $4.26(\mathrm{ABd}, 12.6)$ & - & - \\
\hline 28 & $2.06(\mathrm{~s})$ & $2.06(\mathrm{~s})$ & $1.99(\mathrm{~s})$ & $1.17(\mathrm{~s})$ & $1.82(\mathrm{~s})$ \\
\hline \multirow[t]{2}{*}{ OAc } & $2.03(\mathrm{~s})$ & $2.01(\mathrm{~s})$ & $1.91(\mathrm{~s})$ & $1.86(\mathrm{~s})$ & $2.03(\mathrm{~s})$ \\
\hline & $2.14(\mathrm{~s})$ & $2.07(\mathrm{~s})$ & $2.00(\mathrm{~s})$ & $1.92(\mathrm{~s})$ & $2.15(\mathrm{~s})$ \\
\hline
\end{tabular}

a) Measured in $\mathrm{CDCl}_{3}$. b) Measured in $\mathrm{C}_{5} \mathrm{D}_{5} \mathrm{~N}$. c) Measured in $\mathrm{CDCl}_{3}$ and $\mathrm{CD}_{3} \mathrm{OD}$. d) Recorded in $500 \mathrm{MHz}$. e) Recorded in $400 \mathrm{MHz}$.

of a carbonyl group and the upfield shift of $\mathrm{H}-17(\delta$ $-0.86 \mathrm{ppm}$ ), which was reasonable to assume that $\mathbf{4}$ is the reduction of 16-carbonyl to methylene derivative of $\mathbf{1}$. In the heteronuclear multiple bond correlation (HMBC) spectrum, long-long correlations between H-14 $(\delta$ 1.93, m), H-17 $(\delta$ $1.67, \mathrm{~m}), \mathrm{H}-20(\delta 1.86, \mathrm{~m})$ and $\mathrm{C}-16(\delta 26.3, \mathrm{t})$ were observed, which affirmed the above assumption. Thus, plantagiolide D (3) was assigned to be $(20 S, 22 R)-1 \alpha, 12 \alpha$-diacetoxy$2 \alpha, 3 \alpha ; 6 \alpha, 7 \alpha$-diepoxy-5 $\alpha, 27$-dihydroxy-with-24-enolide.

Plantagiolide D (5), obtained as colorless needles, possesses a molecular formula of $\mathrm{C}_{32} \mathrm{H}_{46} \mathrm{O}_{10}$ (10 degrees of unsaturation) as determined by its (HR)-ESI-MS $\left([\mathrm{M}+\mathrm{Na}]^{+}\right.$, $m / z$ 613.2988) and ${ }^{13} \mathrm{C}-\mathrm{NMR}$ spectrum. The comparison of the spectral data with those of $\mathbf{4}$ revealed that the hydrogen and carbon atom chemical shift values for the rings of $A-D$ in $\mathbf{5}$ are in good agreement with those of $\mathbf{4}$ except for the disappearance of carbon-carbon double bond and a hydroxymethyl group and the existence of one additional oxygenated quaternary carbons $(\delta$ 68.7) and an additional methyl in E-ring. The the heteronuclear multiple bond correlation (HMBC) of H-22 ( $\delta 4.57, \mathrm{dt}, J=3.1,6.3,12.0 \mathrm{~Hz}), \mathrm{H}-25(\delta$ $2.13, \mathrm{~d}, J=7.1 \mathrm{~Hz}), \mathrm{Me}-27(\delta 1.12, \mathrm{~d}, J=7.1 \mathrm{~Hz})$, and Me-28 $(\delta 1.17, \mathrm{~s})$ with $\mathrm{C}-24(\delta 68.7, \mathrm{~s})$ indicated that the additional hydroxyl group was attached to $\mathrm{C}-24$ in compound 5. The doublet at $\delta 0.70(\mathrm{~d}, J=6.9 \mathrm{~Hz})$ suggested that $\mathrm{C}-21$ is a secondary methyl having the usual $\alpha$-orientation, ${ }^{24)}$ which confirmed the large between $\mathrm{H}-17$ and $\mathrm{H}-20(\mathrm{~d}, J=9.1 \mathrm{~Hz})$ and the ROESY correlation of H-20/Me-18 and Me-21/H-12. The identical ${ }^{13} \mathrm{C}$-NMR chemical shifts of $\delta$-lactone moiety of 5 with those of philadelphicalactone $\mathrm{B}^{25)}$ indicated that 5 had the same structure, including stereochemistry, as that of philadelphicalactone $\mathrm{B}$, which $\mathrm{Me}-27$ was $\beta$-eq and $\mathrm{Me}-28$ was $\alpha$-eq and was confirmed via a ROESY experiment. The correlation of $\mathrm{H}-22$ with Me-28, H-25 with Me-28 could be clearly observed from the spectrum, suggesting that the hydroxyl groups located at C-24 and the Me-27 were both in $\beta$-orientation. Therefore, the structure of $\mathbf{5}$ concluded to be $(20 S, 2 R, 24 R, 25 R)-1 \alpha, 12 \alpha$-diacetoxy- $2 \alpha, 3 \alpha ; 6 \alpha, 7 \alpha$ diepoxy-5 $\alpha, 24 \beta$-dihydroxy-withanolide, and designated plantagiolide $\mathrm{D}$.

Plantagiolide E (6) was obtained as colorless needles. The molecular formula, $\mathrm{C}_{32} \mathrm{H}_{46} \mathrm{O}_{11}$, was deduced from the positive high-resolution electrospray ionization mass spectrum (HR-ESI-MS) $\left([\mathrm{M}+\mathrm{Na}]^{+} m / z\right.$ 629.2952) and NMR spectra (Table 2). The comparison of the NMR spectral data with those of 5 showed that the chemical shifts of the rings of $\mathrm{A}$ $\mathrm{D}$ in $\mathbf{6}$ are highly comparable with those of $\mathbf{5}$. The differences were the disappearance of a doublet of Me-27 and the presence of a singlet of $\mathrm{Me}-27$ and the existence of one additional oxygenated quaternary carbons $(\delta$ 75.5) in E-ring. These observations strongly implied that compound $\mathbf{5}$ was the 25-hydroxy derivative of $\mathbf{6}$. Three groups of HMBC correlations were observed to established the structural features of 5: $\mathrm{H}_{2}-23(\delta 1.82 / 2.80, \mathrm{~m})$ showed cross peaks with $\mathrm{C}-24$ $(\delta 72.8, \mathrm{~s}), \mathrm{C}-25(\delta 75.7, \mathrm{~s}) ; \mathrm{Me}-27(\delta 2.05, \mathrm{~s})$ correlated with $\mathrm{C}-24(\delta 72.8, \mathrm{~s}), \mathrm{C}-25(\delta 75.7, \mathrm{~s}) ;$ and Me-28 $(\delta 1.82$, s) correlated with $\mathrm{C}-24(\delta 72.8, \mathrm{~s}), \mathrm{C}-25(\delta 75.7, \mathrm{~s})$. The above observations suggested that the another hydroxyl 
Table $2 .{ }^{13} \mathrm{C}$-NMR Data for Compounds $\mathbf{1}, \mathbf{3}-\mathbf{6}(\delta$ in ppm $)$

\begin{tabular}{|c|c|c|c|c|c|}
\hline Carbon & $\mathbf{1}^{a, d)}$ & $\mathbf{3}^{a, e)}$ & $4^{a, d)}$ & $\mathbf{5}^{c, d)}$ & $6^{b, e)}$ \\
\hline 1 & $71.3 \mathrm{~d}$ & $71.6 \mathrm{~d}$ & $71.5 \mathrm{~d}$ & $71.5 \mathrm{~d}$ & $72.8 \mathrm{~d}$ \\
\hline 2 & $50.8 \mathrm{~d}$ & $51.0 \mathrm{~d}$ & $50.9 \mathrm{~d}$ & $50.8 \mathrm{~d}$ & $52.1 \mathrm{~d}$ \\
\hline 3 & $54.9 \mathrm{~d}$ & $55.0 \mathrm{~d}$ & $54.9 \mathrm{~d}$ & $54.8 \mathrm{~d}$ & $56.0 \mathrm{~d}$ \\
\hline 4 & $32.3 \mathrm{t}$ & $32.5 \mathrm{t}$ & $32.4 \mathrm{t}$ & $32.1 \mathrm{t}$ & $33.5 \mathrm{t}$ \\
\hline 5 & $69.8 \mathrm{~s}$ & $69.9 \mathrm{~s}$ & $69.7 \mathrm{~s}$ & $69.8 \mathrm{~s}$ & $70.8 \mathrm{~s}$ \\
\hline 6 & $56.2 \mathrm{~d}$ & $56.2 \mathrm{~d}$ & $56.0 \mathrm{~d}$ & $55.8 \mathrm{~d}$ & $57.1 \mathrm{~d}$ \\
\hline 7 & $53.5 \mathrm{~d}$ & $53.9 \mathrm{~d}$ & $53.9 \mathrm{~d}$ & $54.0 \mathrm{~d}$ & $54.8 \mathrm{~d}$ \\
\hline 8 & $34.8 \mathrm{~d}$ & $35.6 \mathrm{~d}$ & $35.8 \mathrm{~d}$ & $35.6 \mathrm{~d}$ & $36.6 \mathrm{~d}$ \\
\hline 9 & $28.1 \mathrm{~d}$ & $28.1 \mathrm{~d}$ & $27.8 \mathrm{~d}$ & $27.7 \mathrm{~d}$ & $28.9 \mathrm{~d}$ \\
\hline 10 & $39.8 \mathrm{~s}$ & $39.8 \mathrm{~s}$ & $39.6 \mathrm{~s}$ & $39.4 \mathrm{~s}$ & $40.7 \mathrm{~s}$ \\
\hline 11 & $23.9 \mathrm{t}$ & $24.1 \mathrm{t}$ & $24.4 \mathrm{t}$ & $25.9 \mathrm{t}$ & $25.1 \mathrm{t}$ \\
\hline 12 & $73.6 \mathrm{~d}$ & $75.4 \mathrm{~d}$ & $75.1 \mathrm{~d}$ & $75.2 \mathrm{~d}$ & $76.2 \mathrm{~d}$ \\
\hline 13 & $46.2 \mathrm{~s}$ & $46.0 \mathrm{~s}$ & $45.8 \mathrm{~s}$ & $45.6 \mathrm{~s}$ & $46.5 \mathrm{~s}$ \\
\hline 14 & $39.5 \mathrm{~d}$ & $42.5 \mathrm{~d}$ & $44.1 \mathrm{~d}$ & $44.0 \mathrm{~d}$ & $45.3 \mathrm{~d}$ \\
\hline 15 & $37.1 \mathrm{t}$ & $35.4 \mathrm{t}$ & $22.5 \mathrm{t}$ & $22.3 \mathrm{t}$ & $23.2 \mathrm{t}$ \\
\hline 16 & $215.1 \mathrm{~s}$ & $70.6 \mathrm{~d}$ & $26.3 \mathrm{t}$ & $24.2 \mathrm{t}$ & $26.8 \mathrm{t}$ \\
\hline 17 & $56.0 \mathrm{~d}$ & $49.5 \mathrm{~d}$ & $43.3 \mathrm{~d}$ & $43.1 \mathrm{~d}$ & $44.1 \mathrm{~d}$ \\
\hline 18 & $14.1 \mathrm{q}$ & $13.1 \mathrm{q}$ & $12.2 \mathrm{q}$ & $11.7 \mathrm{q}$ & $12.5 \mathrm{q}$ \\
\hline 19 & $16.1 \mathrm{q}$ & $16.2 \mathrm{q}$ & $16.1 \mathrm{q}$ & $15.8 \mathrm{q}$ & $16.6 \mathrm{q}$ \\
\hline 20 & $34.4 \mathrm{~d}$ & $33.0 \mathrm{~d}$ & $37.9 \mathrm{~d}$ & $38.0 \mathrm{~d}$ & $39.6 \mathrm{~d}$ \\
\hline 21 & $13.1 \mathrm{q}$ & $12.1 \mathrm{q}$ & $11.9 \mathrm{q}$ & $11.3 \mathrm{q}$ & $12.5 \mathrm{q}$ \\
\hline 22 & $77.0 \mathrm{~d}$ & $79.0 \mathrm{~d}$ & $78.3 \mathrm{~d}$ & $77.9 \mathrm{~d}$ & $79.3 \mathrm{~d}$ \\
\hline 23 & $31.6 \mathrm{t}$ & $31.2 \mathrm{t}$ & $29.4 \mathrm{t}$ & $35.2 \mathrm{t}$ & $31.6 \mathrm{t}$ \\
\hline 24 & $152.8 \mathrm{~s}$ & $153.2 \mathrm{~s}$ & $152.8 \mathrm{~s}$ & $68.7 \mathrm{~s}$ & $72.8 \mathrm{~s}$ \\
\hline 25 & $125.7 \mathrm{~s}$ & $125.7 \mathrm{~s}$ & $125.6 \mathrm{~s}$ & $45.9 \mathrm{~d}$ & $75.7 \mathrm{~s}$ \\
\hline 26 & $166.3 \mathrm{~s}$ & $166.6 \mathrm{~s}$ & $166.5 \mathrm{~s}$ & $175.0 \mathrm{~s}$ & $176.4 \mathrm{~s}$ \\
\hline 27 & $56.8 \mathrm{t}$ & $57.0 \mathrm{t}$ & $57.0 \mathrm{t}$ & $8.7 \mathrm{q}$ & $19.2 \mathrm{q}$ \\
\hline 28 & $20.1 \mathrm{q}$ & $20.1 \mathrm{q}$ & $20.1 \mathrm{q}$ & $27.2 \mathrm{q}$ & $24.2 \mathrm{q}$ \\
\hline \multirow[t]{4}{*}{ OAc } & $170.0 \mathrm{~s}$ & $169.9 \mathrm{~s}$ & $169.8 \mathrm{~s}$ & $170.1 \mathrm{~s}$ & $171.1 \mathrm{~s}$ \\
\hline & $170.1 \mathrm{~s}$ & $170.1 \mathrm{~s}$ & $169.9 \mathrm{~s}$ & $170.3 \mathrm{~s}$ & $171.1 \mathrm{~s}$ \\
\hline & $19.8 \mathrm{q}$ & $20.2 \mathrm{q}$ & $19.9 \mathrm{q}$ & $19.8 \mathrm{q}$ & $20.8 \mathrm{q}$ \\
\hline & $21.2 \mathrm{q}$ & $21.3 \mathrm{q}$ & $21.2 \mathrm{q}$ & $20.8 \mathrm{q}$ & $21.5 \mathrm{q}$ \\
\hline
\end{tabular}

a) Measured in $\mathrm{CDCl}_{3}$. b) Measured in $\mathrm{C}_{5} \mathrm{D}_{5} \mathrm{~N}$. c) Measured in $\mathrm{CDCl}_{3}$ and $\mathrm{CD}_{3} \mathrm{OD}$. d) Recorded in $125 \mathrm{MHz}$. e) Recorded in $100 \mathrm{MHz}$.

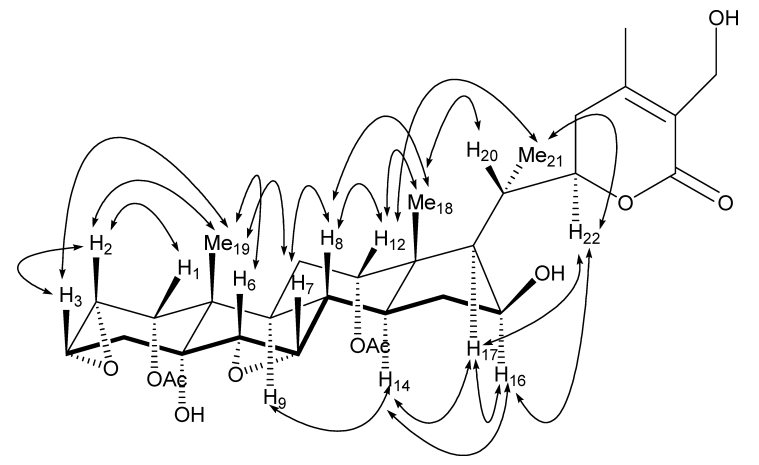

Fig. 1. Significant ROESY Correlations of Plantagiolide B (2)

group should connect with $\mathrm{C}-25$. The ROESY cross-peaks of $\mathrm{H}-22 / \mathrm{Me}-27, \mathrm{H}-22 / \mathrm{Me}-28$ established the Me-27 was $\alpha$-ax and Me-28 was in $\alpha$-eq, as drawn. Accordingly, the structure of plantagiolide E (6) concluded to be $(20 S, 22 R, 24 R, 25 R)$ $1 \alpha, 12 \alpha$-diacetoxy- $2 \alpha, 3 \alpha ; 6 \alpha, 7 \alpha$-diepoxy- $5 \alpha, 24 \beta, 25 \beta$-trihydroxy-withanolide.

The five new withanolides $\mathbf{1}-\mathbf{6}$ obtained from this plant were tested for in vitro cytotoxicity against Eca-109, SPC-A1, BGC-823, AGS, K562 cells. However, all of them were noncytotoxic.

\section{Experimental}

General Procedues Melting points were recorded on an XRC-1 micro melting point apparatus and are uncorrected. Optical rotations were measured in a JASCO DIP-370 digital polarimeter. UV spectra were measured using a Shimadzu UV-2401PC spectrophotometer. IR spectra were obtained on a Bio-Rad FTS-135 infrared spectrophotometer with $\mathrm{KBr}$ pellets. FAB mass spectrum were obtained on a VG Auto spec-3000 spectrometer and high-resolution ESI mass spectrum were recorded on an API Qstar Pulsar instrument. 1D and 2D NMR experiments were performed on Bruker AM400 and DRX-500 instruments with TMS as internal standard. Chemical shifts $(\delta)$ were expressed in ppm with reference to the solvent signals. Column chromatography was performed on silica gel (200-300 mesh, Qingdao Marine Chemical Inc., Qingdao, People's Republic of China) or on silica gel $\mathrm{H}(10-40 \mu \mathrm{m}$, Qingdao Marine Chemical Inc.) and Lichroprep Rp-18 (43- $63 \mu \mathrm{m}$, Merck). Fractions were monitored by TLC and spots were visualized by heating silica gel plates sprayed with $10 \% \mathrm{H}_{2} \mathrm{SO}_{4}$ in EtOH.

Plant Material The whole plant Tacca plantaginea were collected in Guilin, Guangxi Zhuang Autonomous Region, People's Republic of China, in August 1999, and identified by professor De-Ding Tao (Kunming Institute of Botany, the Chinese Academy of Sciences).

Extraction and Isolation The powdered air-dried plant of $T$. plantaginea $(30 \mathrm{~kg})$ were exhaustively extracted three times with $95 \% \mathrm{EtOH}$ under reflux. After evaporated, the resulting residue $(1.5 \mathrm{~kg})$ was extracted with $\mathrm{CHCl}_{3}$ and $n$-butanol, successively. The $\mathrm{CHCl}_{3}$ layer $(700 \mathrm{~g})$ was subjected to silica gel column chromatography eluting with a petroleum ether-AcOEt gradient $(1: 0,10: 1,5: 1,7: 3,1: 1)$ to give five fractions $1-$ 5. Fraction $4(150 \mathrm{~g})$ was repeatedly chromatographed over silica gel $\left(\mathrm{CHCl}_{3} / \mathrm{H}_{2} \mathrm{O}\right)$ and $\mathrm{RP}-18\left(\mathrm{MeOH} / \mathrm{H}_{2} \mathrm{O}\right)$ to afforded $1(70 \mathrm{mg}), 3(33 \mathrm{mg}), 4$ $(25 \mathrm{mg}), \mathbf{5}(120 \mathrm{mg})$, and $\mathbf{6}(11 \mathrm{mg})$. The EtOAc layer $(50 \mathrm{~g})$ was repeatedly chromatographed on silica gel column, eluting with a $\mathrm{CHCl}_{3}-\mathrm{MeOH}$ gradient $(9: 1)$ to give compound $\mathbf{2}(120 \mathrm{mg})$.

Plantagiolide A (1): Colorless needles from chloroform. mp 246-247 ${ }^{\circ} \mathrm{C}$. $[\alpha]_{\mathrm{D}}^{26}+11.1^{\circ}\left(c=3.3, \mathrm{CHCl}_{3}\right)$. UV $\lambda_{\max }\left(\mathrm{CHCl}_{3}\right) \mathrm{nm}(\log \varepsilon): 241$ (3.96). IR (KBr) $v_{\max } \mathrm{cm}^{-1}: 3480,2978,2938,1736,1706,1651,1466,1421,1380$ 1246, 1027. ${ }^{1} \mathrm{H}-\mathrm{NMR}\left(\mathrm{CDCl}_{3}, 500 \mathrm{MHz}\right)$ and ${ }^{13} \mathrm{C}-\mathrm{NMR}\left(\mathrm{CDCl}_{3}, 125 \mathrm{MHz}\right)$ see Tables 1 and 2. Positive FAB-MS $m / z: 603[\mathrm{M}+\mathrm{H}]^{+}, 543[\mathrm{M}-$ $\left.\mathrm{CH}_{3} \mathrm{COOH}\right]^{+}, 525\left[\mathrm{M}-\mathrm{CH}_{3} \mathrm{COOH}-\mathrm{H}_{2} \mathrm{O}\right]^{+}, 483\left[\mathrm{M}-2 \times \mathrm{CH}_{3} \mathrm{COOH}\right]^{+}$, $465\left[\mathrm{M}-2 \times \mathrm{CH}_{3} \mathrm{COOH}-\mathrm{H}_{2} \mathrm{O}\right]^{+}, 447\left[\mathrm{M}-2 \times \mathrm{CH}_{3} \mathrm{COOH}-2 \times \mathrm{H}_{2} \mathrm{O}\right]^{+}$. Positive HR-ESI-MS $m / z$ : $625.2623[\mathrm{M}+\mathrm{Na}]^{+}\left(\mathrm{Calcd}\right.$ for $\mathrm{C}_{32} \mathrm{H}_{42} \mathrm{O}_{11} \mathrm{Na}$, 625.2624).

Plantagiolide B (3): White amorphous powder. $m p 253-255^{\circ} \mathrm{C} .[\alpha]_{\mathrm{D}}^{26}$ $+65.2^{\circ}\left(c=2.6, \mathrm{CHCl}_{3}\right)$. UV $\lambda_{\text {max }}\left(\mathrm{CHCl}_{3}\right) \mathrm{nm}(\log \varepsilon): 241$ (3.90). IR $(\mathrm{KBr})$ $v_{\max } \mathrm{cm}^{-1}: 3443,2927,1734,1635,1379,1250,1031 .{ }^{1} \mathrm{H}-\mathrm{NMR}\left(\mathrm{CDCl}_{3}\right.$, $400 \mathrm{MHz})$ and ${ }^{13} \mathrm{C}-\mathrm{NMR}\left(\mathrm{CDCl}_{3}, 400 \mathrm{MHz}\right)$ see Tables 1 and 2. Positive FAB-MS $m / z: 605[\mathrm{M}+\mathrm{H}]^{+}$. Positive HR-ESI-MS $m / z: 627.2776[\mathrm{M}+\mathrm{Na}]^{+}$ (Calcd for $\mathrm{C}_{32} \mathrm{H}_{44} \mathrm{O}_{11} \mathrm{Na}, 627.2781$ ).

Plantagiolide $\mathrm{C}(4)$ : Colorless needles from chloroform. $\mathrm{mp} 208-210^{\circ} \mathrm{C}$. $[\alpha]_{\mathrm{D}}^{26}+93.5^{\circ}\left(c=4.2, \mathrm{CHCl}_{3}\right)$. UV $\lambda_{\max }\left(\mathrm{CHCl}_{3}\right) \mathrm{nm}(\log \varepsilon): 240(3.88)$. IR (KBr) $v_{\max } \mathrm{cm}^{-1}: 3448,2944,1732,1705,1641,1397,1381,1252,1030$. ${ }^{1} \mathrm{H}-\mathrm{NMR}\left(\mathrm{CDCl}_{3}, 500 \mathrm{MHz}\right)$ and ${ }^{13} \mathrm{C}-\mathrm{NMR}\left(\mathrm{CDCl}_{3}, 125 \mathrm{MHz}\right)$ see Tables 1 and 2. Positive FAB-MS $m / z$ : $589[\mathrm{M}+\mathrm{H}]^{+}$. Positive HR-ESI-MS $m / z$ : $611.2835[\mathrm{M}+\mathrm{Na}]^{+}\left(\right.$Calcd for $\left.\mathrm{C}_{32} \mathrm{H}_{44} \mathrm{O}_{10} \mathrm{Na}, 611.2832\right)$.

Plantagiolide D (5): Colorless needles from chloroform-methanol. $\mathrm{mp}$ $237-239^{\circ} \mathrm{C} .[\alpha]_{\mathrm{D}}^{26}+66.1^{\circ}\left(c=13.6, \mathrm{CHCl}_{3}\right)$. IR $(\mathrm{KBr}) v_{\max } \mathrm{cm}^{-1}: 3497$, 2982, 1739, 1690, 1400, 1382, 1261, 1239. ${ }^{1} \mathrm{H}-\mathrm{NMR}\left(\mathrm{CDCl}_{3}-\mathrm{CD}_{3} \mathrm{OD}\right.$, $400 \mathrm{MHz})$ and ${ }^{13} \mathrm{C}$-NMR $\left(\mathrm{CDCl}_{3}-\mathrm{CD}_{3} \mathrm{OD}, 100 \mathrm{MHz}\right)$ see Tables 1 and 2. Positive FAB-MS $m / z: 591[\mathrm{M}+\mathrm{H}]^{+}, 573\left[\mathrm{M}+\mathrm{H}-\mathrm{H}_{2} \mathrm{O}\right]^{+}, 531[\mathrm{M}+\mathrm{H}-$ $\left.\mathrm{CH}_{3} \mathrm{COOH}\right]^{+}, 513\left[\mathrm{M}+\mathrm{H}-\mathrm{H}_{2} \mathrm{O}-\mathrm{CH}_{3} \mathrm{COOH}\right]^{+}, 495\left[\mathrm{M}+\mathrm{H}-2 \times \mathrm{H}_{2} \mathrm{O}-\right.$ $\left.\mathrm{CH}_{3} \mathrm{COOH}\right]^{+}, 471\left[\mathrm{M}+\mathrm{H}-2 \times \mathrm{CH}_{3} \mathrm{COOH}\right]^{+}$. Positive HR-ESI-MS $\mathrm{m} / \mathrm{z}$ : $613.3005[\mathrm{M}+\mathrm{Na}]^{+}\left(\right.$Calcd for $\left.\mathrm{C}_{32} \mathrm{H}_{46} \mathrm{O}_{10} \mathrm{Na}, 613.2988\right)$.

Plantagiolide E (6): Colorless needles from methanol. mp $328-3300^{\circ} \mathrm{C}$. $[\alpha]_{\mathrm{D}}^{26}+198.5^{\circ}\left(c=2.7\right.$, pyridine). IR (KBr) $v_{\max } \mathrm{cm}^{-1}: 3479,3456,2944$, $1736,1693,1381,1257,1030 .{ }^{1} \mathrm{H}-\mathrm{NMR}$ (pyridine- $d_{5}, 400 \mathrm{MHz}$ ) and ${ }^{13} \mathrm{C}-$ NMR (pyridine- $d_{5}, 100 \mathrm{MHz}$ ) see Tables 1 and 2. Positive FAB-MS $m / z: 607$ $[\mathrm{M}+\mathrm{H}]^{+}$. Positive HR-ESI-MS $m / z: 629.2952[\mathrm{M}+\mathrm{Na}]^{+}$(Calcd for $\mathrm{C}_{32} \mathrm{H}_{46} \mathrm{O}_{11} \mathrm{Na}$, 629.2937).

\section{References}

1) Delectis Florae Reipublicae Popularis Sinicae Agendae Academiae Sinicae Edita, "Flora Republicae Popularis Sinicae, Tomus 16," Science Press, Beijing, 1985, p. 42.

2) Muhlbauer A., Gehling M., Velten R., Andersh W., Erdelen C., Harder A., Marczok P., Nauen R., Turber A., Tran V. S., Adam G., Liu J. K., Int. Pat. Wo 01/04256 to Bayer AG Germany, 2001.

3) Huang Y., Liu J. K., Mühlbauer A., Henkel T., Helv. Chim. Acta, 85, 2553-2558 (2002).

4) Mühlbauer A., Seip S., Nowak A., Tran V. S., Helv. Chim. Acta, 86, 2065-2072 (2003). 
5) Tripathi R. D., Tiwari K. P., Planta Med., 41, 414-415 (1981).

6) Yokosuka A., Mimaki Y., Sakagami H., Sashida Y., J. Nat. Prod., 65, 283-289 (2002).

7) Ngok F. K., Kelginbaev A. N., Gorvits M. B., Abubakirov N. K., Chem. Nat. Compd., 16, 262-264 (1980).

8) Zhou J., Chen C. X., Liu R. M., Yang C. R., Acta Botanica Sinica, 25 $568-573$ (1983).

9) Abedel-Aziz A., Brain K., Blunden G., Grabb T., Bashir A. K., Phytochemistry, 29, 1643-1646 (1990).

10) Abedel-Aziz A., Brain K., Blunden G., Grabb T., Bashir A. K., Planta Med., 56, 218-221 (1990).

11) Yokosuka A., Mimaki Y., Sashida Y., J. Nat. Prod., 65, 1293-1298 (2002).

12) Yokosuka A., Mimaki Y., Sashida Y., Phytochemistry, 61, 73-78 (2002).

13) Yokosuka A., Mimaki Y., Sashida Y., J. Nat. Prod., 66, 876-878 (2003).

14) Yokosuka A., Mimaki Y., Sashida Y., Chem. Pharm. Bull., 52, 13961398 (2004).

15) Yokosuka A., Mimaki Y., Sakuma C., Sashida Y., Steroids, 70, 257265 (2005).
16) Jiangsu New Medical College, "The Dictionary of Traditional Chinese Medinces," Shanghai Science and Technology Press, Shanghai, 1977, p. 524.

17) Chen Z. L., Wang B. D., Chen M. Q., Tetrahedron Lett., 28, $1673-$ 1676 (1987).

18) Chen Z. L., Wang B. D., Shen J. H., Phytochemistry, 27, 2999-3001 (1988).

19) Shen J. H., Chen Z. L., Gao Y. S., Chin. J. Chem., 1, $92-94$ (1991).

20) Shen J. H., Chen Z. L., Gao Y. S., Phytochemistry, 42, 891-893 (1996).

21) Chen Z. L., Shen J. H., Gao Y. S., Wichtl M., Planta Med., 63, 40-43 (1997).

22) Tinley T. L., Randall-Hlubek D. A., Leal R. M., Jackson E. M., Cessac J. W., Quada J. C., Hemscheidt T. K., Mooberry S. L., Cancer Res., 63, $3211-3220$ (2003).

23) Liu H. Y., Chen C. X., Chin. Chem. Lett., 13, 633-636 (2002).

24) Miera L., Lal P., Sangwan R. S., Sangwan N. S., Uniyal G. C., Tuli R., Phytochemistry, 66, 2702-2707 (2005)

25) Su B. N., Misico R., Park E. J., Santarsiero B. D., Mesecar A. D., Fong H. H. S., Pezzuto J. M., Kinghorn A. D., Tetrahedron, 58, 3453-3466 (2002). 